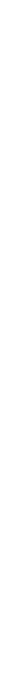

\title{
Parámetros e indicadores que determinan una agricultura eficiente - Parameters and Indicators that determine efficient Agriculture
}

\author{
Authors: $\quad$ Angel Martinez T, Sol Rodriguez R. \\ Submitted: $\quad$ 15. May 2020 \\ Published: $\quad$ 16. May 2020 \\ Volume: 7 \\ Issue: 3 \\ Affiliation: $\quad$ RUDN University, Moscow, Russia \\ Languages: Spanish, Castilian \\ Keywords: $\quad$ Agriculture, Production, Efficient / Agricultura, Producción, \\ Eficiencia. \\ Categories: Life Sciences \\ DOI: $\quad$ 10.17160/josha.7.3.673
}

\section{Abstract:}

The article studies the theoretical aspects that allow us to identify when an agricultural company is obtaining a good production and specifies the main economic, social and environmental indicators that we must take into account when we decide to invest in agriculture. 


\title{
Parameters and indicators that determine efficient agriculture - Parámetros e indicadores que determinan una agricultura eficiente
}

By Angel F. Martinez T, Sol A. Rodriguez R.

Peoples' Friendship University of Russia, RUDN University

Moscow, Russia.

\begin{abstract}
The article studies the theoretical aspects that allow us to identify when an agricultural company is obtaining a good production and specifies the main economic, social and environmental indicators that we must take into account when we decide to invest in agriculture.
\end{abstract}

Keywords: Agriculture, Production, Efficient / Agricultura, Producción, Eficiencia. 
La eficiencia de una empresa agrícola se ve reflejada en su producción, la cual está conectada con el interés económico que determina el propósito de la producción. La eficacia refleja el grado de éxito que se ha logrado en relación con la producción social e individual. El resultado de cualquier actividad de las empresas agrícolas se caracteriza a través del efecto económico. El resultado final de este indicador se ve afectado por muchos parámetros reflejados en las dependencias entre los niveles de gestión, la afiliación del sector y otros vínculos. La eficiencia del funcionamiento de cualquier empresa agrícola está determinada no solo por la relación del resultado financiero teniendo en cuenta los recursos gastados, así como ciertos criterios e indicadores.

El criterio de eficiencia debe reflejar el objetivo inmediato y tener en cuenta las peculiaridades de las condiciones en las que la empresa realiza sus propias actividades en la industria. En estos criterios de eficiencia económica se establecen los ingresos brutos y netos, capacidad de fondos, costo de producción y comercial, beneficio y rentabilidad. Al formar una evaluación del desempeño de cualquier empresa agrícola, estos criterios son los principales indicadores de una economía estable y exitosa en una organización.

Para evaluar la efectividad de la actividad de una empresa agrícola desde todos los aspectos, es necesario tener en cuenta no solo los criterios de funcionamiento de la empresa, sino también los sistemas de indicadores. Los indicadores de rendimiento deben reflejar el contenido del proceso de producción del sistema investigado y sus subsistemas, en este caso la producción agrícola, el ciclo del capital y los bienes invertidos, la capacidad de recursos de la empresa, la capacidad de utilizarlos, intercambiarlos, consumirlos, distribuirlos y obtener el máximo beneficio, teniendo en cuenta la capacidad de la organización.

Los principales indicadores que caracterizan a una empresa agrícola como eficiente pueden reflejarse en dos partes interconectadas como son los indicadores privados y generalizados. 
Los indicadores privados se caracterizan por ser los aspectos específicos del proceso de producción en el que se utilizan ciertos recursos, como el costo necesario para la producción. Los indicadores generalizados están influenciados por los indicadores privados del sistema y estos determinan los gastos generales realizados fuera de la empresa como los impuestos, el transporte etc.

El sistema de rendimiento examinado ofrece la oportunidad de abordar la selección de los grupos de indicadores pertinentes y darles una justificación y de esta manera garantizar que la empresa genere ingresos y no egresos determinando el desempeño de cada unidad individual o empresa en su conjunto. La eficiencia se caracteriza por un conjunto de indicadores que reflejan el grado de participación de ciertos recursos en el proceso de producción de productos agrícolas.

Según el estudio tecnológico de la eficiencia, los principales indicadores de esta son el rendimiento de los cultivos, la productividad, la intensidad energética en la producción, la producción bruta de la agricultura con precios competitivos y el aumento de la producción bruta. Los Indicadores económicos que nos permiten determinar el éxito de nuestra producción son el margen bruto, el costo comercial, el costo del producto, recuperación del capital invertido, la sostenibilidad financiera y solvencia de la empresa junto con la rentabilidad.

Las leyes de muchos países exigen una evaluación del impacto ecológico y, como consecuencia es necesario tener en cuenta de que forma afectamos el medio ambiente y cuál es el costo que nuestra empresa debe pagar para reducir el impacto, los principales indicadores que debemos identificar son la posibilidad de reducir la contaminación del medio ambiente por cualquier medio, el mantenimiento de la sostenibilidad ambiental y el impacto medioambiental que produce nuestra empresa. 
También debemos tener en cuenta los principales indicadores del criterio de rendimiento social como el consumo del trabajador promedio anual, la remuneración, los indicadores demográficos para la reproducción de la población beneficiad, el consumo actual por persona, los ingresos reales totales y los ingresos reales por costo de vida.

Cada uno de estos indicadores están entrelazados entre sí por eso es necesario hacer un estudio e identificar cuáles de estos influyen significativamente en nuestra producción y de esta manera es posible determinar la rentabilidad de nuestra empresa en el sector agrícola, un error de cálculo de estos factores puede ocasionar grandes perdidas en nuestra inversión y como consecuencia la perdida de nuestro capital. Por esta razón se recomienda antes de invertir en el sector agrícola, hacer un esquema que identifique los indicadores potenciales que generaran gastos en nuestra inversión para ser tomados en cuenta en el costo final del producto.

\section{Bibliography:}

1) García Suárez E. ; García Arias A. ; Vázquez González I. (2019) Current productive situation of cattle farms in Spain: The Cantabrian Cornice case. Economía Agraria y Recursos Naturales - Agricultural and Resource Economics, [S.1.], v. 19, n. 2, p. 93111. Recuperado desde: //polipapers.upv.es/index.php/EARN/article/view/earn.2019.02.05

2) Economía agraria y recursos naturales - agecon serch / https://ageconsearch.umn.edu/search? $\ln =$ en $\& c c=1380 \& p=\& f=\&$ action_search=Bus $\underline{\mathrm{car}}$

3) Opazo J. (2018). Eficiencia y competitividad en la agricultura - La huerta digital blog. Recuperado desde: / https://lahuertadigital.es/eficiencia-y-competitividad-enagricultura/

4) Olivares Fernández J. (2012). Indicadores de sostenibilidad de la agricultura y ganadería españolas - Plataforma Tecnólogica de Agricultura Sostenible-PTASRecuperado desde: https://www.interempresas.net/Agricola/Articulos/103498$\underline{\text { Indicadores-de-sostenibilidad-de-la-agricultura-y-ganaderia-espanolas.html }}$ 
About the author:

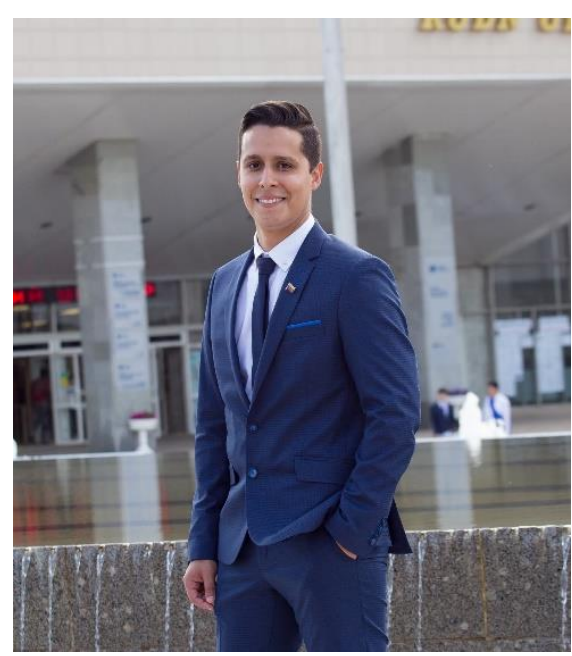

Civil Engineer from the National Experimental Polytechnic University of the FANB in Venezuela, Master in Residential, Public and Industrial Architecture in the Russian Federation, with experience as analyst and supervisor of construction works, author of agricultural production projects (Vertical Farms) through the design of buildings with intelligent and renewable architecture and technology making use of the benefits of hydroponics as a basis for production 